\title{
A Summary of Meteorological Parameters During Space Shuttle Pad Exposure Periods
}

\author{
B. Glemn Overbey" \\ Raytheon, Huntsville, AL,35805 \\ and \\ Barry C. Roberts ${ }^{\dagger}$ \\ NASA Marshall Space Flight Center, Huntsville, AL, 35812
}

\begin{abstract}
During the 113 missions of the Space Transportation System (STS), the Space Shuttle neet has been exposed to the elements on the launch pad for a total of 4195 days. The Natural Environments Branch at Marshall Space Flight Center archives atmospheric environments to which the Space Shuttle vehicles are exposed. This paper provides a summary of the historical record of the meteorological conditions encountered by the Space Shuttle fleet during the pad exposure period. Sources of the surface parameters, including temperature, dew point temperature, relative humidity, wind speed, wind direction, sea level pressure and precipitation are presented. Data is provided from the first launch of the STS in 1981 through the launch of STS-107 in 2003.
\end{abstract}

\section{Nomenclature}

$\bar{H} \quad=$ scale height

$P_{\Delta Z} \quad=$ barometric pressure at height $\Delta Z$ above sea level

$S L P \quad=$ sea level pressure

$\Delta Z \quad=$ height above sea level

\section{Introduction}

FROM the time the Space Shuttle stack is rolled out from the Vehicle Assembly Building (VAB) to the launch I pad, it is exposed to the natural environment at Kennedy Space Center (KSC). From the first mission, STS-1 which launched on April 12, 1981, thru STS-107, which launched on January 16, 2003, the Space Shuttle vehicles have been exposed on the launch pad for a total of 4195 days.

As part of the Columbia Accident Investigation, the Natural Environments Branch at Marshall Space Flight Center (MSFC) was tasked to document the meteorological conditions to which all the Space Shuttle vehicles were exposed while on the launch pad. The intent of this paper is to give a description of the meteorological data that has been recorded while the Space Shuttle vehicle was on the launch pad. A National Aeronautics and Space Administration (NASA) Technical Memorandum is planned to be released in 2005 that contains a full set of pad exposure period meteorological data for all of the Space Shuttle missions.

\section{MSFC Natural Environments Branch}

The Natural Environments Branch is part of the Spacecraft \& Vehicle Systems Department at Marshall Space Flight Center. The mission of the group is to provide information on naturally occurring space environments, terrestrial environments, and planetary atmospheric environments to customers at MSFC and other NASA centers. The Natural Environment Branch's Terrestrial and Planetary Environments Team archives and models the terrestrial environment to support aerospace vehicle design, development, and analysis and models planetary environments to be used for aerospace vehicle engineering studies.

\footnotetext{
- Principal Physics Engineer, Information Technology \& Scientific Services, AIAA Member.

${ }^{\dagger}$ Flight Vehicle Atmospheric Environments, Natural Environments Branch, EV13, AIAA Member.
} 
The Terrestrial and Planetary Environments Team has been tasked by the Space Shuttle program to provide technical management of the natural environments area. This includes certifying new atmospheric instrumentation for use by the Space Shuttle program, archiving surface and upper atmospheric thermodynamic and wind measurements made at the Kennedy Space Center and the U.S. Air Force Eastern Range, climatological modeling, day-of-launch wind profile monitoring and verification, documentation of atmospheric environment during ascent (the final meteorological profile), and terrestrial environment requirements development.

\section{Description of Data Sources}

The data in this paper were collected over a period of approximately 23 years at surface observation stations and from meteorological (MET) towers at KSC.

\section{A. Surface Observations}

Hourly surfaces observations from two sites are included in the archive and used for this paper. Table 1 gives the source information, including the station call sign, Weather Bureau Army/Navy (WBAN) designation, World Meteorological Organization (WMO) designation, approximate location with respect to the Space Shuttle Launch Complex 39 (LC-39) and period of record for the archive. The location of these observation stations is shown in Figure 1.

Due to questionable hourly precipitation data from Station 12868 , daily precipitation totals as measured at Station 12886 and provided by the Air Force Combat Climatology Center are presented in this document through STS-86 (September 1997). Since STS-86, precipitation has been measured near the pads with optical rain gauges.

The surface observation data were obtained near ground level. Temperature, relative humidity, pressure and precipitation are measured at approximately $1.5 \mathrm{~m}$ above ground level. Wind speed and direction are measured at approximately $9 \mathrm{~m}$ above ground level.

\section{B. MET Tower Observations}

Data from six MET towers have been archived by the MSFC Natural Environments Branch and are used in this document. Tower 393 is on the northwest side of Launch Pad 39A (LP39A) and Tower 394 is on the southeast side. Tower 397 is on the northwest side of LP-39B and Tower 398 is on the southeast side. The location of these towers is shown in Figure 1. Each tower is approximately $0.4 \mathrm{~km}$ from their respective launch pad.

The wind data from Towers 393, 394, 397 and 398 were measured at $18.3 \mathrm{~m}$ above natural grade (ANG). The temperature, humidity and dew point

Table 1. Surface observation data source descriptions.

\begin{tabular}{|l|l|l|}
\hline \multicolumn{1}{|c|}{ Source } & Location & \multicolumn{1}{|c|}{$\begin{array}{c}\text { Period of } \\
\text { Record }\end{array}$} \\
\hline $\begin{array}{l}\text { Cape Canaveral Air Force } \\
\text { Station (CCAFS) } \\
\text { Call Sign: XMR } \\
\text { WBAN Station \#12868 }\end{array}$ & $\begin{array}{l}16 \mathrm{~km} \\
\text { south of } \\
\text { LC-39 }\end{array}$ & $\begin{array}{l}12 / 1980- \\
11 / 1984\end{array}$ \\
\hline $\begin{array}{l}\text { Shuttle Landing Facility } \\
\text { (SLF) }\end{array}$ & $\begin{array}{l}8 \mathrm{~km} \\
\text { west of } \\
\text { Call Signs: X68, TTS \& } \\
\text { KTTS } \\
\text { WMO Station \#74794 } \\
\text { WBAN Station \#12886 }\end{array}$ & $\begin{array}{l}12 / 1984- \\
\text { Present }\end{array}$ \\
\hline
\end{tabular}
from Towers 393, 394, 397 and 398 were

measured at both $1.8 \mathrm{~m}$ ANG which is equivalent to approximately $6.4 \mathrm{~m}$ above mean sea level (MSL) and $18.3 \mathrm{~m}$ ANG. All data from Tower 313 were measured at $16 \mathrm{~m}$ ANG because Tower 313 is not instrumented at $18.3 \mathrm{~m}$ ANG.

From STS-56 (March 1993) to STS-80 (October 1996), the tower data consisted of temperature, dew point, relative humidity, wind speed and wind direction. After this time, pressure measurements were added to Towers 394 and 398 only. The pressure data were measured at approximately $6.4 \mathrm{~m}$ above MSL. The measured pressure values were adjusted to sea level pressure to provide a common reference level using the following equation:

$$
S L P=P_{\Delta Z}\left(1+\frac{\Delta Z}{\bar{H}}\right)
$$


Where, $S L P$ is sea level pressure, $P_{\Delta Z}$ is the measured (barometric) pressure, $\Delta Z$ is the height of the barometer above sea level in meters and $\bar{H}$ is the scale height $(8500 \mathrm{~m})$. This equation is a simplified form of the hypsometric equation ${ }^{1}$ and is accurate to within about $0.1 \mathrm{hPa}$ for $\triangle Z$ less than about $120 \mathrm{~m}$.

After STS-80, optical rain gauges were added to Towers 393, 394, 397 and 398. The optical rain gauges provide one-second precipitation measurements. This data is available only sporadically prior to STS-87 (October 1987).

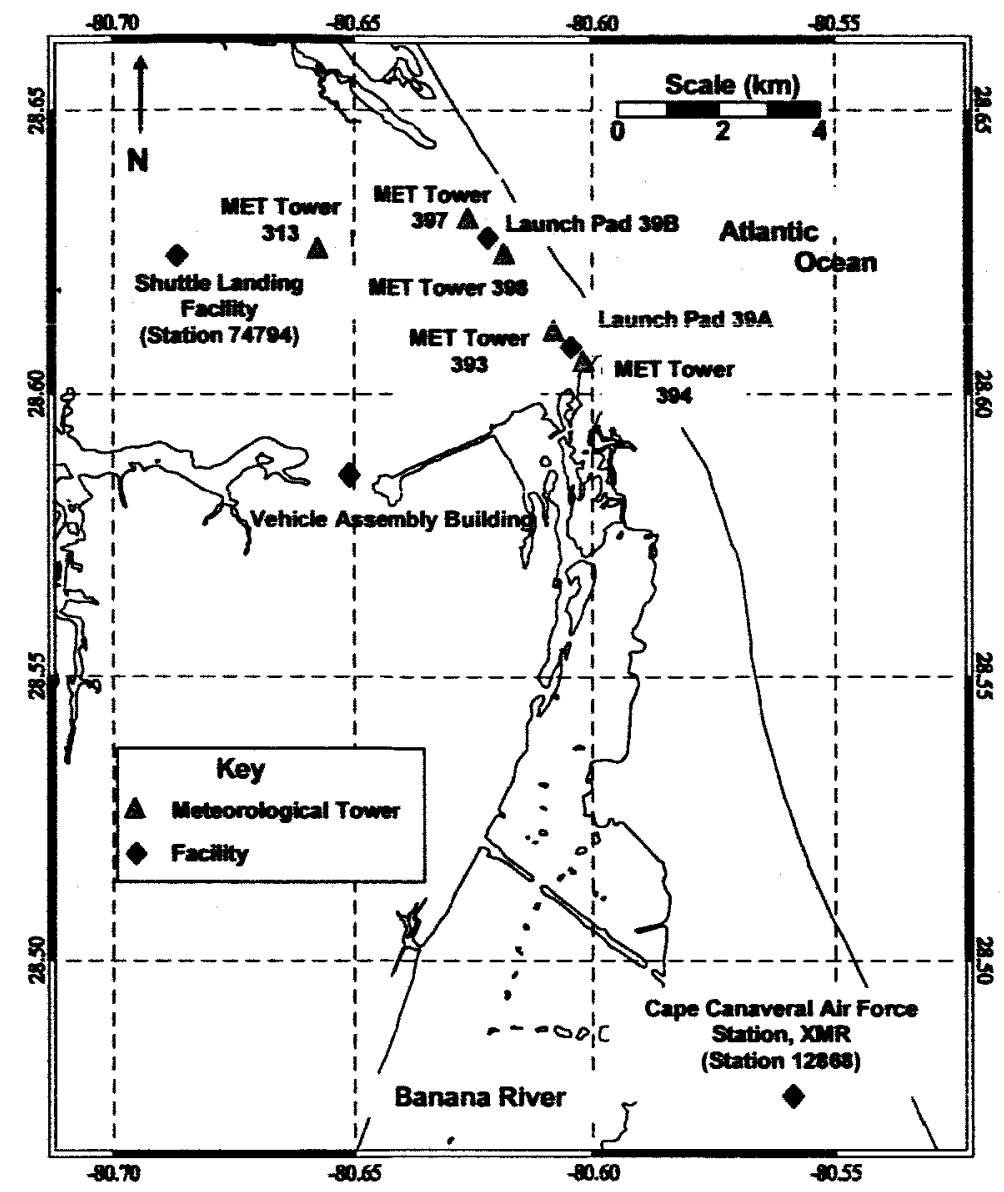

Figure 1. Kennedy Space Center meteorological observation locations.

\section{Sample Data}

\section{A. STS-113}

This section presents an example of the type of data available for all Space Shuttle missions. This data is from STS-113, launched on November 23, 2002. Table 2 shows the hourly extremes of the various meteorological parameters while STS-113 was on the pad. Figures 2 through 7 show the temperature, relative humidity, wind speed, wind direction, sea level pressure, and precipitation as recorded at MET Tower 394 while STS-113 was on the pad. 
Table 2. STS-113 pad exposure period hourly extremes.

\begin{tabular}{|l|c|}
\hline Min. Temperature & $6.7^{\circ} \mathrm{C}\left(44^{\circ} \mathrm{F}\right)$ \\
\hline Max. Temperature & $30.0^{\circ} \mathrm{C}\left(86^{\circ} \mathrm{F}\right)$ \\
\hline Min. Relative Humidity & $35 \%$ \\
\hline Max. Relative Humidity & $100 \%$ \\
\hline Min. Sea Level Pressure & $1004.7 \mathrm{hPa}(29.67 \mathrm{in} \mathrm{Hg})$ \\
\hline Max. Sea Level Pressure & $1028.4 \mathrm{hPa}(30.37 \mathrm{in} \mathrm{Hg})$ \\
\hline Max. Wind Speed \& & $17.5 \mathrm{~m} / \mathrm{s}(34 \mathrm{kts})$ \\
Associated Wind Direction & $269 \mathrm{deg}$. \\
\hline Total Precipitation & $58.7 \mathrm{~mm}(2.31 \mathrm{in})$ \\
\hline
\end{tabular}

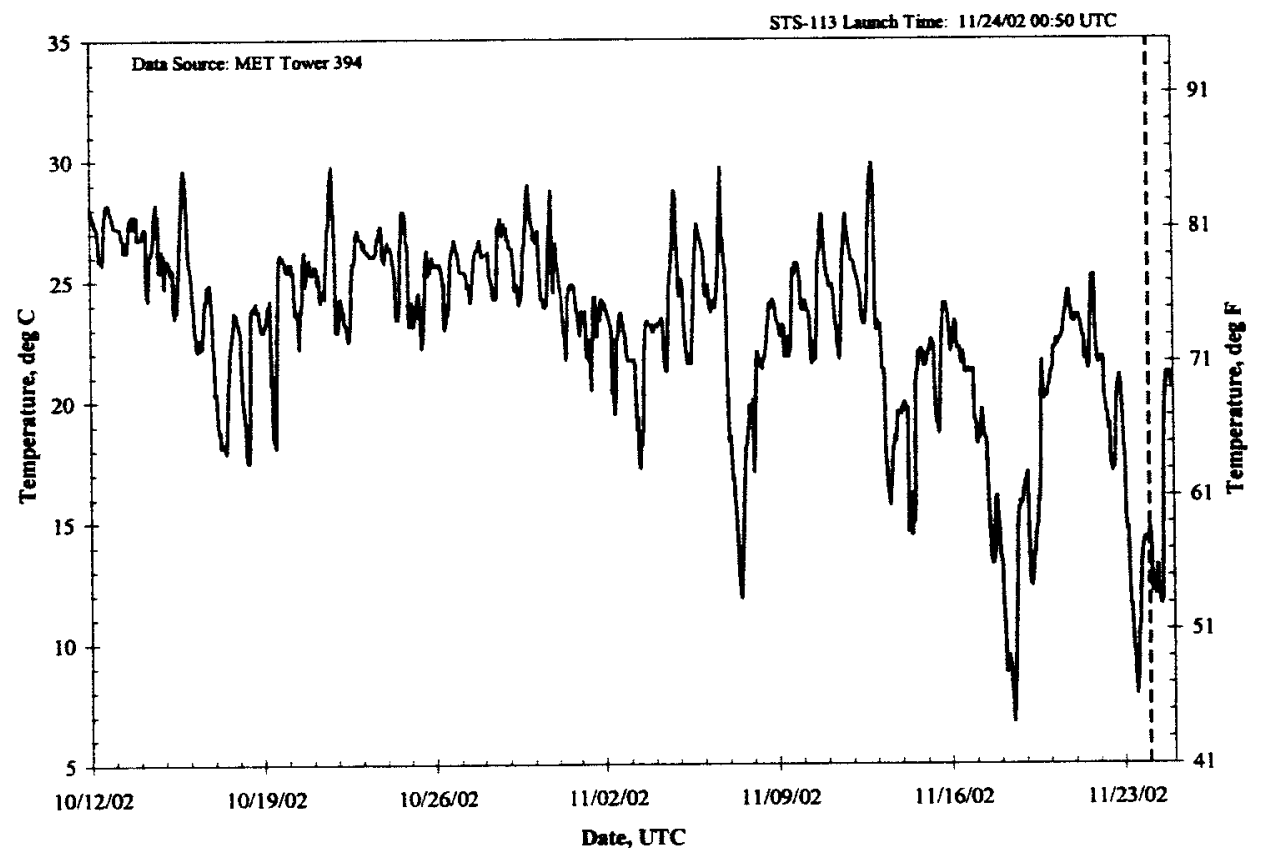

Figure 2. STS-113 Hourly temperature at $18 \mathrm{~m}$ (60 ft.). 


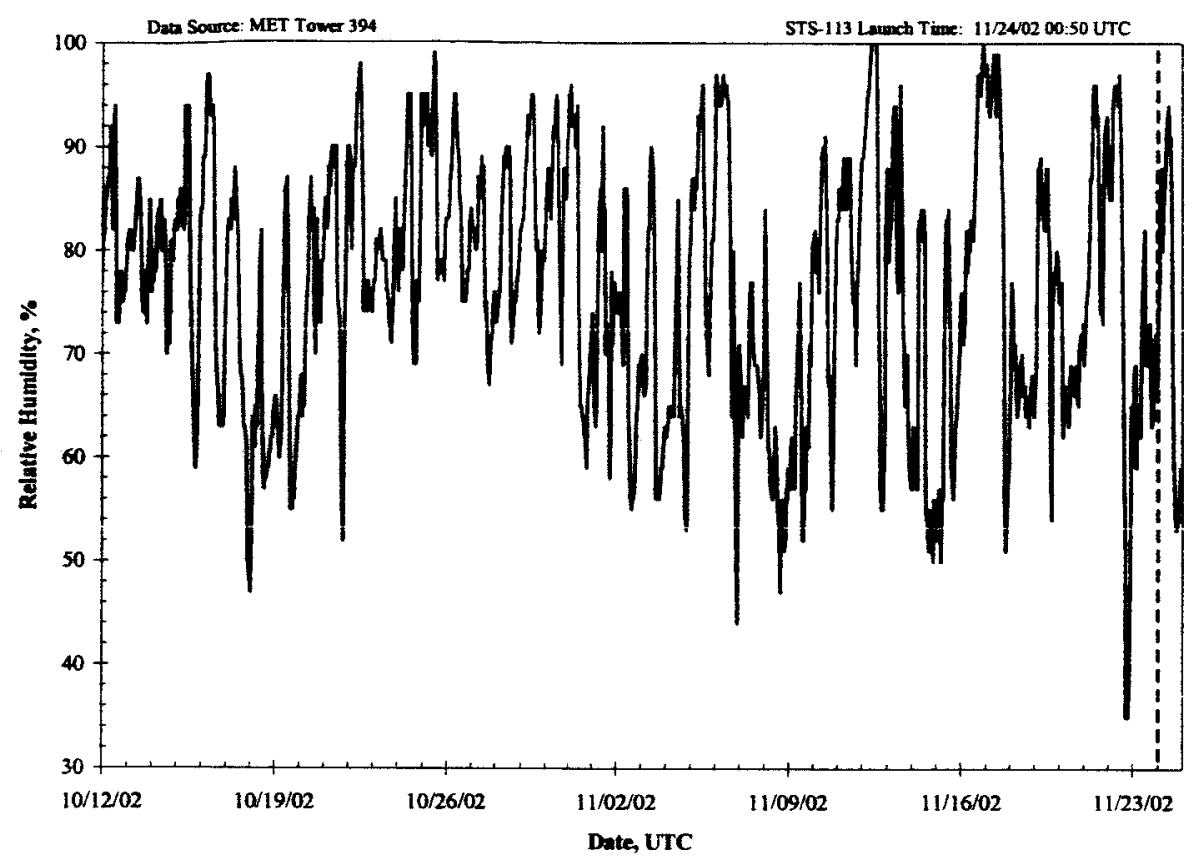

Figure 3. STS-113 Hourly relative humidity at $18 \mathrm{~m}(60 \mathrm{ft}$.$) .$

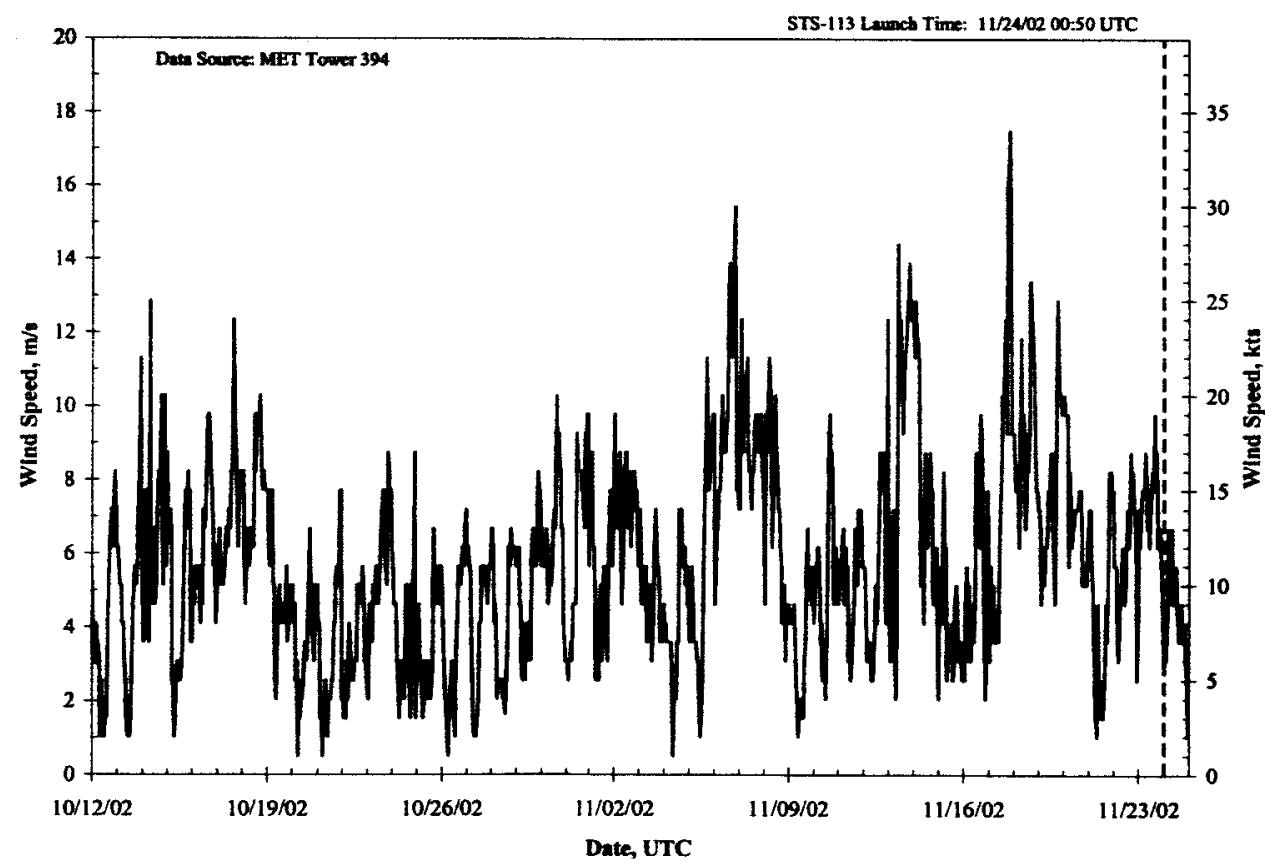

Figure 4. STS-113 Hourly wind speed at $18 \mathrm{~m}$ (60 ft.). 


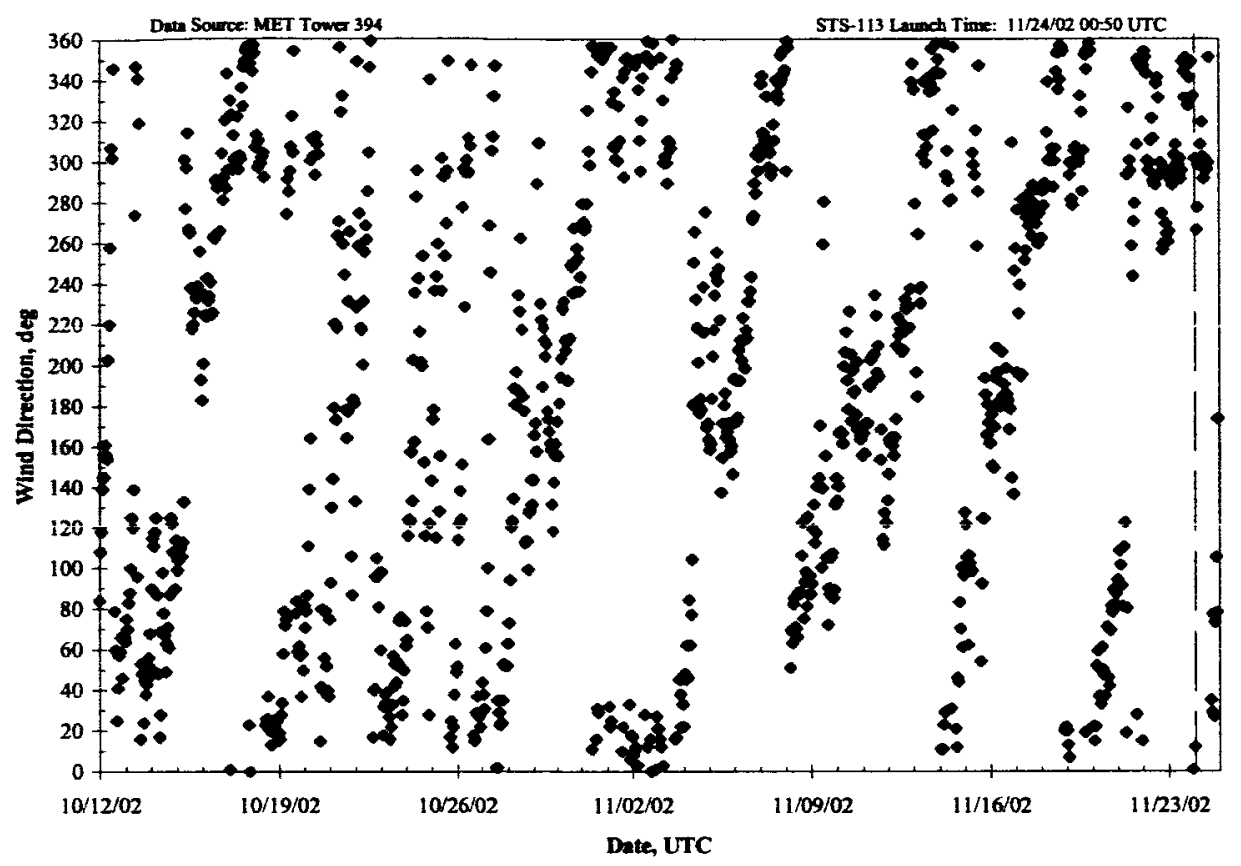

Figure 5. STS-113 Hourly wind direction at $18 \mathrm{~m}$ (60 ft.).

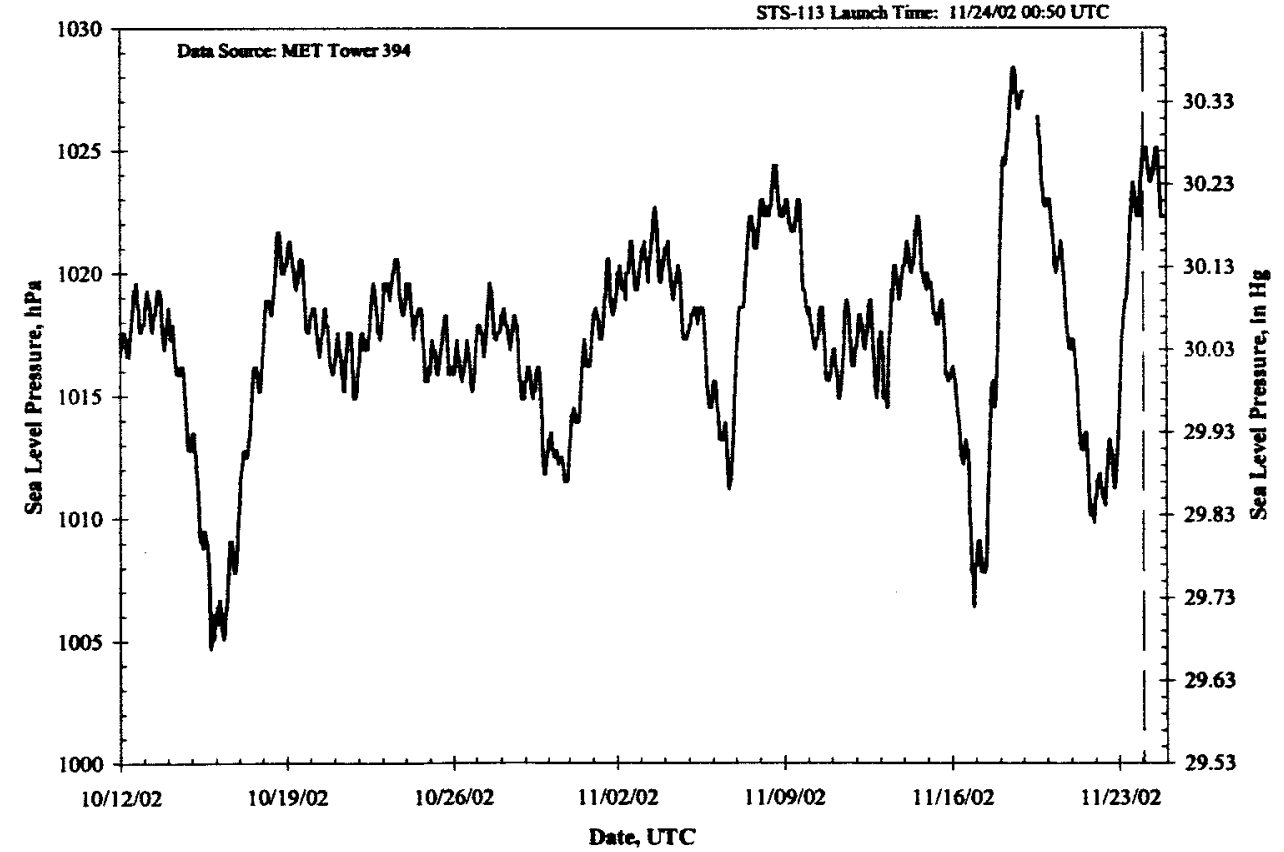

Figure 6. STS-113 Hourly sea level pressure. 


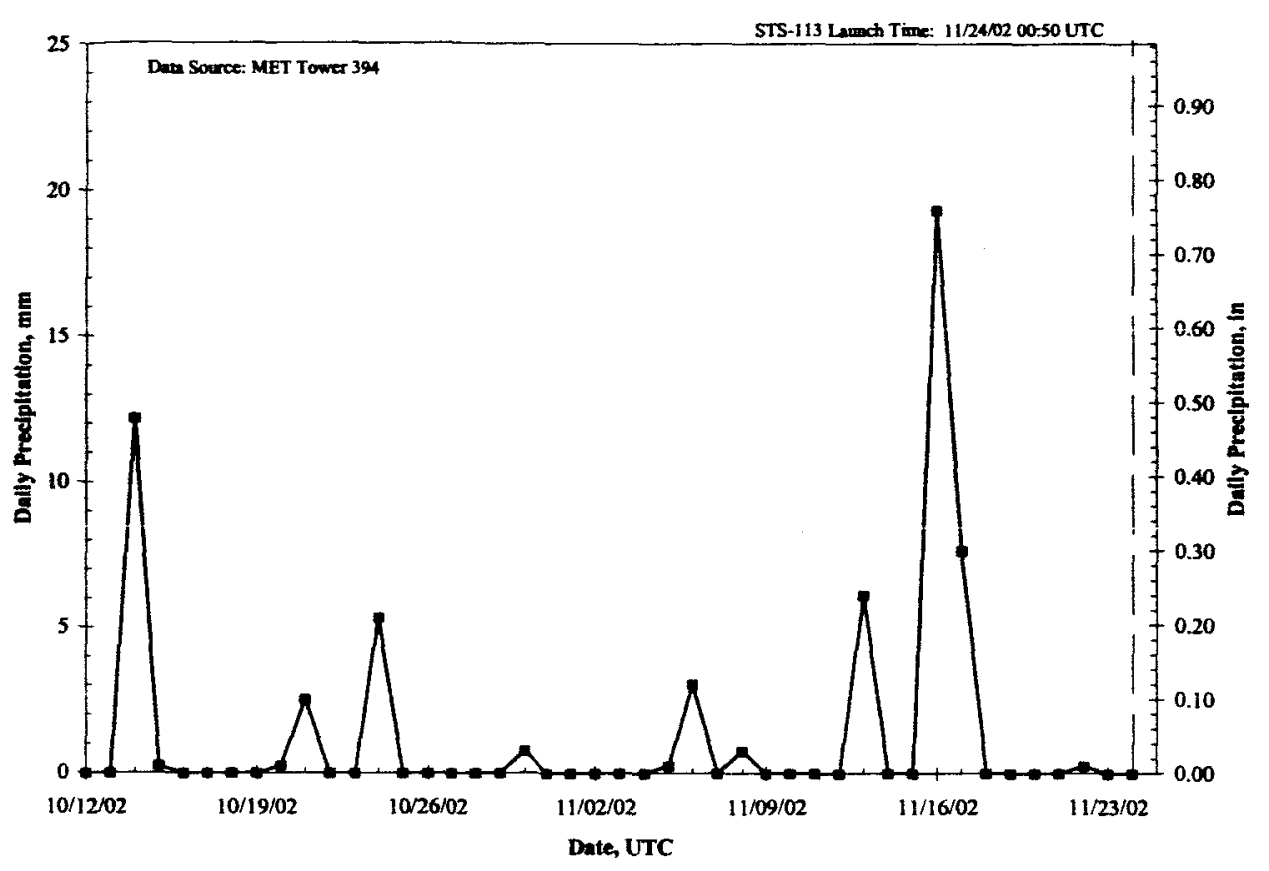

Figure 7. STS-113 Daily precipitation totals.

B. T-0 Meteorological Data Summary

Table 3 presents a summary of the T- 0 (liftoff) meteorological conditions for each of the Space Shuttle missions. Data presented includes temperature, relative humidity, wind speed, and wind direction.

Table 3. T-0 (liftoff) meteorological conditions for all Space Shuttle missions.

\begin{tabular}{|c|c|c|c|c|c|c|c|}
\hline Mission & Launch Date & $\begin{array}{c}\text { Time } \\
\text { (UTC) }\end{array}$ & $\begin{array}{c}\text { Temperature } \\
\left({ }^{\circ} \mathrm{C}\right)\end{array}$ & $\begin{array}{c}\text { Relative } \\
\text { Hurnidity } \\
(\%)\end{array}$ & $\begin{array}{c}\text { Sea Level Pressure } \\
(\mathrm{hPa})\end{array}$ & $\begin{array}{c}\text { Wind Speed } \\
(\mathrm{m} / \mathrm{s})\end{array}$ & $\begin{array}{c}\text { Wind Direction } \\
(\mathrm{deg})\end{array}$ \\
\hline STS-1 & 1981.04 .12 & $12: 00$ & 21.1 & 82 & 1023.7 & 3.6 & 125 \\
\hline STS-2 & 1981.11 .12 & $15: 10$ & 22.8 & 61 & 1016.9 & 8.2 & 345 \\
\hline STS-3 & 1982.03 .22 & $16: 00$ & 24.4 & 71 & 1016.6 & 2.1 & 050 \\
\hline STS-4 & 1982.06 .27 & $15: 00$ & 29.1 & 70 & 1020.7 & 1.8 & 133 \\
\hline STS-5 & 1982.11 .11 & $12: 19$ & 22.0 & 59 & 1023.3 & 6.7 & 090 \\
\hline STS-6 & 1983.04 .04 & $18: 30$ & 22.8 & 55 & 1019.0 & 3.9 & 063 \\
\hline STS-7 & 1983.06 .18 & $11: 33$ & 25.1 & 80 & 1015.2 & 1.8 & 010 \\
\hline STS-8 & 1983.08 .30 & $06: 32$ & 23.9 & 97 & 1011.9 & 2.7 & 269 \\
\hline STS-9 & 1983.11 .28 & $16: 00$ & 24.4 & 83 & 1015.9 & 5.8 & 183 \\
\hline $41-B$ & 1984.02 .03 & $13: 00$ & 16.7 & 75 & 1018.0 & 0.0 & 000 \\
\hline $41-C$ & 1984.04 .06 & $13: 58$ & 15.8 & 56 & 1015.6 & 6.5 & 320 \\
\hline $41-D$ & 1984.08 .30 & $12: 42$ & 26.2 & 81 & 1018.0 & 0.9 & 106 \\
\hline $41-G$ & 1984.10 .05 & $11: 03$ & 23.3 & 60 & 1021.7 & 5.0 & 073 \\
\hline
\end{tabular}


Table 3. (continued)

\begin{tabular}{|c|c|c|c|c|c|c|c|}
\hline Mission & Launch Date & $\begin{array}{l}\text { Time } \\
\text { (UTC) }\end{array}$ & $\begin{array}{l}\text { Temperature } \\
\left({ }^{\circ} \mathrm{C}\right)\end{array}$ & $\begin{array}{c}\text { Relative } \\
\text { Humidity } \\
\text { (\%) }\end{array}$ & $\begin{array}{c}\text { Sea Level Pressure } \\
\qquad(\mathrm{hPa})\end{array}$ & $\begin{array}{l}\text { Wind Speed } \\
\qquad(\mathrm{m} / \mathrm{s})\end{array}$ & $\begin{array}{l}\text { Wind Direction } \\
\text { (deg) }\end{array}$ \\
\hline $51-\mathrm{A}$ & 1984.11 .08 & $12: 15$ & 19.9 & 64 & 1023.4 & 7.0 & 024 \\
\hline $51-C$ & 1985.01 .24 & $19: 50$ & 17.7 & 46 & 1018.0 & 5.2 & 228 \\
\hline 51-D & 1985.04 .12 & $13: 59$ & 20.7 & 55 & 1026.4 & 6.1 & 082 \\
\hline 51-B & 1985.04 .29 & $16: 02$ & 27.4 & 65 & 1013.5 & 3.5 & 005 \\
\hline $51-G$ & 1985.06.17 & 11:33 & 22.8 & 91 & 1020.7 & 0.9 & 201 \\
\hline $51-F$ & 1985.07 .29 & $21: 00$ & 28.3 & 72 & 1018.0 & 4.5 & 101 \\
\hline $51-1$ & 1985.08 .27 & $10: 58$ & 24.3 & 86 & 1023.0 & 4.3 & 073 \\
\hline $51-\mathrm{J}$ & 1985.10 .03 & 15:15 & 28.2 & 79 & 1019.3 & 5.2 & 213 \\
\hline $61-A$ & 1985.10 .30 & $17: 00$ & 27.8 & 72 & 1006.4 & 3.9 & 217 \\
\hline $61-\mathrm{B}$ & 1985.11 .27 & $00: 29$ & 22.7 & 81 & 1020.9 & 3.1 & 165 \\
\hline $61-C$ & 1986.01 .12 & $11: 55$ & 12.0 & 84 & 1021.3 & 4.7 & 323 \\
\hline $51-\mathrm{L}$ & 1986.01 .28 & $16: 38$ & 2.6 & 27 & 1026.1 & 6.1 & 331 \\
\hline STS-26 & 1988.09 .29 & $15: 37$ & 28.9 & 56 & 1019.0 & 4.2 & 058 \\
\hline STS-27 & 1988.12.02 & $14: 31$ & 13.9 & 50 & 1027.8 & 7.8 & 314 \\
\hline STS-29 & 1989.03 .13 & 14:57 & 17.8 & 78 & 1019.3 & 5.2 & 242 \\
\hline STS-30 & 1989.05 .04 & $18: 47$ & 26.1 & 57 & 1020.0 & 6.6 & 106 \\
\hline STS-28 & 1989.08 .08 & $12: 37$ & 26.7 & 80 & 1011.9 & 3.8 & 252 \\
\hline STS-34 & 1989.10 .18 & $16: 54$ & 30.0 & 52 & 1015.2 & 4.1 & 193 \\
\hline STS-33 & 1989.11.23 & $00: 23$ & 18.9 & 80 & 1013.2 & 5.2 & 208 \\
\hline STS-32 & 1990.01 .09 & $12: 35$ & 12.2 & 100 & 1020.7 & 2.1 & 246 \\
\hline STS-36 & 1990.02 .28 & 07:50 & 18.3 & 71 & 1026.8 & 7.2 & 072 \\
\hline STS-31 & 1990.04 .24 & $12: 34$ & 22.2 & 63 & 1020.0 & 5.7 & 080 \\
\hline STS-41 & 1990.10 .06 & $11: 47$ & 27.2 & 73 & 1018.3 & 7.2 & 090 \\
\hline STS-38 & 1990.11 .15 & $23: 48$ & 21.1 & 63 & 1025.7 & 8.8 & 084 \\
\hline STS-35 & 1990.12 .02 & $06: 49$ & 21.7 & 61 & 1024.0 & 6.6 & 088 \\
\hline STS-37 & 1991.04 .05 & $14: 23$ & 22.8 & 84 & 1025.6 & 5.7 & 074 \\
\hline STS- 39 & 1991.04 .28 & $11: 33$ & 22.2 & 95 & 1014.9 & 3.9 & 191 \\
\hline STS-40 & 1991.06 .05 & $13: 25$ & 23.9 & 83 & 1020.0 & 2.1 & 234 \\
\hline STS-43 & 1991.08 .02 & 15:02 & 27.8 & 73 & 1018.6 & 5.2 & 170 \\
\hline STS-48 & 1991.09 .12 & $23: 11$ & 25.0 & 71 & 1014.6 & 3.8 & 074 \\
\hline STS-44 & 1991.11 .24 & $23: 44$ & 12.2 & 48 & 1019.6 & 2.6 & 301 \\
\hline STS -42 & 1992.01 .22 & $14: 53$ & 17.2 & 86 & 1022.0 & 3.1 & 038 \\
\hline STS-45 & 1992.03.24 & $13: 13$ & 18.9 & 72 & 1018.0 & 7.2 & 359 \\
\hline STS-49 & 1992.05 .07 & $23: 40$ & 17.2 & 70 & 1013.5 & 4.1 & 322 \\
\hline STS-50 & 1992.06 .25 & $16: 12$ & 28.3 & 72 & 1013.2 & 6.9 & 151 \\
\hline STS-46 & 1992.07.31 & $13: 57$ & 28.9 & 76 & 1020.3 & 3.2 & 310 \\
\hline STS -47 & 1992.09 .12 & $14: 23$ & 27.8 & 70 & 1020.1 & 5.0 & 044 \\
\hline STS-52 & 1992.10 .22 & $17: 10$ & 25.0 & 49 & 1027.7 & 8.4 & 048 \\
\hline STS-53 & 1992.12 .02 & $13: 24$ & 11.1 & 87 & 1020.0 & 3.1 & 215 \\
\hline STS-54 & 1993.01 .13 & $13: 59$ & 21.1 & 94 & 1022.0 & 2.9 & 180 \\
\hline STS-56 & 1993.04 .08 & $05: 29$ & 18.3 & 74 & 1017.3 & 5.1 & 065 \\
\hline STS-55 & 1993.04 .26 & $14: 50$ & 25.0 & 70 & 1019.2 & 6.2 & 173 \\
\hline
\end{tabular}


Table 3. (continued)

\begin{tabular}{|c|c|c|c|c|c|c|c|}
\hline Mission & Launch Date & $\begin{array}{l}\text { Time } \\
\text { (UTC) }\end{array}$ & $\begin{array}{c}\text { Temperature } \\
\left({ }^{\circ} \mathrm{C}\right)\end{array}$ & $\begin{array}{c}\text { Relative } \\
\text { Humidity } \\
(\%)\end{array}$ & $\begin{array}{c}\text { Sea Level Pressure } \\
(\mathrm{hPa})\end{array}$ & $\begin{array}{l}\text { Wind Speed } \\
\qquad(\mathrm{m} / \mathrm{s})\end{array}$ & $\begin{array}{c}\text { Wind Direction } \\
\text { (deg) }\end{array}$ \\
\hline STS-57 & 1993.06 .21 & $13: 07$ & 28.3 & 65 & 1019.1 & 3.1 & 091 \\
\hline STS-51 & 1993.09 .12 & $11: 45$ & 22.8 & 98 & 1020.0 & 2.0 & 294 \\
\hline STS-58 & 1993.10 .18 & $14: 53$ & 25.6 & 72 & 1017.3 & 3.2 & 316 \\
\hline STS-61 & 1993.12 .02 & 09:26 & 18.9 & 84 & 1024.4 & 1.8 & 070 \\
\hline STS-60 & 1994.02 .03 & $12: 10$ & 7.2 & 82 & 1027.8 & 4.2 & 318 \\
\hline STS-62 & 1994.03 .04 & $13: 53$ & 11.7 & 57 & 1018.3 & 5.0 & 285 \\
\hline STS-59 & 1994.04 .09 & 11:05 & 22.0 & 81 & 1024.0 & 9.8 & 100 \\
\hline STS-65 & 1994.07 .08 & $16: 43$ & 30.1 & 72 & 1023.4 & 4.7 & 100 \\
\hline STS-64 & 1994.09 .09 & $22: 23$ & 28.9 & 75 & 1016.9 & 3.8 & 111 \\
\hline STS-68 & 1994.09 .30 & $11: 16$ & 25.8 & 75 & 1016.7 & 5.2 & 070 \\
\hline STS-66 & 1994.11 .03 & $17: 00$ & 24.4 & 65 & 1022.4 & 5.2 & 065 \\
\hline STS-63 & 1995.02 .03 & $05: 22$ & 13.2 & 83 & 1018.7 & 5.2 & 239 \\
\hline STS-67 & 1995.03 .02 & $06: 38$ & 17.6 & 87 & 1014.0 & 4.8 & 257 \\
\hline STS-71 & 1995.06 .27 & 19:32 & 29.6 & 83 & 1016.0 & 4.0 & 089 \\
\hline STS-70 & 1995.07 .13 & $13: 42$ & 28.4 & 80 & 1016.7 & 2.7 & 051 \\
\hline STS-69 & 1995.09 .07 & $15: 09$ & 28.9 & 77 & 1011.9 & 3.3 & 160 \\
\hline STS-73 & 1995.10 .20 & $13: 53$ & 25.1 & 99 & 1012.6 & 2.8 & 297 \\
\hline STS-74 & 1995.11 .12 & $12: 31$ & 9.8 & 82 & 1019.0 & 5.0 & 301 \\
\hline STS-72 & 1996.01 .11 & $09: 41$ & 4.9 & 87 & 1020.1 & 4.2 & 290 \\
\hline STS-75 & 1996.02 .22 & $20: 18$ & 22.7 & 78 & 1014.6 & 2.9 & 064 \\
\hline STS-76 & 1996.03 .22 & 08:13 & 7.3 & 88 & 1016.0 & 3.8 & 253 \\
\hline STS-77 & 1996.05 .19 & $10: 30$ & 21.3 & 95 & 1018.7 & 0.8 & 239 \\
\hline STS-78 & 1996.06 .20 & 14:49 & 28.4 & 77 & 1015.6 & 0.8 & 266 \\
\hline STS-79 & 1996.09 .16 & 08:55 & 26.0 & 86 & 1014.0 & 4.2 & 153 \\
\hline STS-80 & 1996.11 .19 & $19: 56$ & 25.1 & 49 & 1011.9 & 3.2 & 240 \\
\hline STS-81 & 1997.01 .12 & $09: 27$ & 16.2 & 79 & 1020.1 & 4.3 & 343 \\
\hline STS-82 & 1997.02 .11 & 08:55 & 11.1 & 94 & 1021.4 & 3.3 & 299 \\
\hline STS-83 & 1997.04 .04 & $19: 21$ & 23.7 & 50 & 1021.4 & 4.5 & 127 \\
\hline STS-84 & 1997.05 .15 & 08:08 & 21.2 & 82 & 1015.3 & 2.5 & 300 \\
\hline STS-94 & 1997.07 .01 & 18:02 & 28.7 & 74 & 1014.0 & 4.6 & 035 \\
\hline STS-85 & 1997.08 .07 & $14: 41$ & 28.3 & 81 & 1017.4 & 2.8 & 020 \\
\hline STS-86 & 1997.09 .26 & $02: 34$ & 26.2 & 92 & 1010.6 & 3.4 & 188 \\
\hline STS-87 & 1997.11.19 & $19: 46$ & 20.2 & 69 & 1020.6 & 6.4 & 358 \\
\hline STS-89 & 1998.01 .23 & $02: 48$ & 20.0 & 89 & 1016.7 & 7.9 & 149 \\
\hline STS-90 & 1998.04 .17 & 18:19 & 27.0 & 63 & 1018.0 & 6.3 & 144 \\
\hline STS-91 & 1998.06 .02 & $22: 06$ & 35.4 & 54 & 1009.6 & 5.3 & 232 \\
\hline STS-95 & 1998.10.29 & $19: 20$ & 24.9 & 53 & 1015.2 & 2.9 & 016 \\
\hline STS-88 & 1998.12 .04 & 08:36 & 22.2 & 83 & 1020.1 & 3.4 & 133 \\
\hline STS-96 & 1999.05 .27 & $10: 50$ & 20.9 & 96 & 1016.7 & 2.9 & 235 \\
\hline STS-93 & 1999.07 .23 & 04:31 & 25.6 & 89 & 1017.3 & 1.4 & 233 \\
\hline STS-103 & 1999.12 .20 & $00: 50$ & 18.9 & 74 & 1016.7 & 2.7 & 312 \\
\hline STS-99 & 2000.02 .11 & $17: 43$ & 19.3 & 60 & 1020.7 & 5.5 & 155 \\
\hline
\end{tabular}


Table 3. (continued)

\begin{tabular}{|c|c|c|c|c|c|c|c|}
\hline Mission & Launch Date & $\begin{array}{c}\text { Time } \\
(\text { UTC) }\end{array}$ & $\begin{array}{c}\text { Temperature } \\
\left({ }^{\circ} \mathrm{C}\right)\end{array}$ & $\begin{array}{c}\text { Relative } \\
\text { Humidity } \\
(\%)\end{array}$ & $\begin{array}{c}\text { Sea Level Pressure } \\
(\mathrm{hPa})\end{array}$ & $\begin{array}{c}\text { Wind Speed } \\
(\mathrm{m} / \mathrm{s})\end{array}$ & $\begin{array}{c}\text { Wind Direction } \\
\text { (deg) }\end{array}$ \\
\hline STS-101 & 2000.05 .19 & $10: 11$ & 22.2 & 77 & 1022.4 & 3.4 & 128 \\
\hline STS-106 & 2000.09 .08 & $12: 46$ & 27.2 & 80 & 1014.6 & 4.1 & 008 \\
\hline STS-92 & 2000.10 .11 & $23: 17$ & 22.8 & 67 & 1020.6 & 6.8 & 050 \\
\hline STS-97 & 2000.12 .01 & $03: 06$ & 17.2 & 65 & 1022.4 & 3.9 & 007 \\
\hline STS-98 & 2001.02 .07 & $23: 13$ & 18.3 & 83 & 1024.7 & 2.7 & 059 \\
\hline STS-102 & 2001.03 .08 & $11: 42$ & 5.6 & 71 & 1018.6 & 3.6 & 289 \\
\hline STS-100 & 2001.04 .19 & $18: 41$ & 20.6 & 38 & 1026.1 & 3.3 & 076 \\
\hline STS-104 & 2001.07 .12 & $09: 04$ & 24.7 & 95 & 1014.6 & 4.0 & 218 \\
\hline STS-105 & 2001.08 .10 & $21: 10$ & 29.1 & 76 & 1016.6 & 4.9 & 103 \\
\hline STS-108 & 2001.12 .05 & $22: 19$ & 23.2 & 80 & 1023.4 & 7.2 & 052 \\
\hline STS-109 & 2002.03 .01 & $11: 22$ & 15.6 & 73 & 1025.7 & 6.3 & 066 \\
\hline STS-110 & 2002.04 .08 & $20: 44$ & 24.4 & 51 & 1023.4 & 7.9 & 131 \\
\hline STS-111 & 2002.06 .05 & $21: 23$ & 28.9 & 73 & 1015.2 & 4.9 & 105 \\
\hline STS-112 & 2002.10 .07 & $19: 46$ & 28.3 & 69 & 1013.5 & 6.4 & 020 \\
\hline STS-113 & 2002.11 .24 & $00: 50$ & 13.9 & 75 & 1024.0 & 2.7 & 004 \\
\hline STS-107 & 2003.01 .16 & $15: 39$ & 18.3 & 68 & 1024.0 & 0.8 & 173 \\
\hline
\end{tabular}

\section{Conclusion}

A summary of the types of meteorological data recorded during Space Shuttle pad exposure periods has been presented. A NASA Technical Memorandum with a full set of data for each Space Shuttle mission is being prepared for publication in 2005. All the data referenced in this paper are available from the Natural Environments Branch at Marshall Space Flight Center.

\section{Acknowledgments}

The authors wish to thank Ms. Lee Murray of United Space Alliance at Kennedy Space Center and Ms. Kathy Winters of the 45th Weather Squadron at Cape Canaveral Air Force Station who were instrumental in obtaining source data. This work was conducted under contract NAS8-00187.

\section{References}

'Wallace, J.M., and Hobbs, P.V., Atmospheric Science An Introductory Survey, Academic Press, San Diego, 1977,467 pp. 